\title{
The Counterjet in the Nucleus of Centaurus A
}

\author{
R. A. Preston, S. J. Tingay, D.L. Jones, D.W. Murphy, \& D.L. Meier \\ Jet Propulsion Laboratory, Caltech, Pasadena, CA 91109, U.S.A.
}

D.L. Jauncey, J.E. Reynolds, \& A.K. Tzioumis CSIRO, Australia Telescope National Facility, NSW 2121, Australia

Lovell, J.E.J. ${ }^{1}$, McCulloch, P.M., \& Costa, M.E. University of Tasmania, Hobart, Tasmania 7001, Australia

G. Nicolson

Hartebeesthoek Radio Astronomy Observatory, Krugersdorp, South Africa

\begin{abstract}
We have observed the nucleus of Centaurus A with the VLBA and the SHEVE arrays, individually and in combination. In sensitive experiments at $8.4 \mathrm{GHz}$ which use both arrays together we have detected components in a sub-parsec-scale counterjet. The counterjet is also seen in 2.3 and $22 \mathrm{GHz}$ images. The nuclear region appears to be partially obscured by a free-free absorbing structure of about $1 \mathrm{pc}$ in extent. The jet axis appears to lie at a $50^{\circ}-70^{\circ}$ angle to our line of sight.
\end{abstract}

\section{The Nature of the Nuclear Region}

The images reveal that the nucleus of Centaurus A consists of a compact core with a jet containing embedded components extending to the northeast (Jauncey et al. 1995, Tingay et al. 1996, Preston et al. 1996). To the southwest of the core are two weak components which are presumably the brightest knots in the counterjet (Jones et al. 1996). The counterjet knots are visible closest to the core at the highest frequency $(22 \mathrm{GHz})$ and farthest from the core at the lowest frequency $(2.3 \mathrm{GHz})$, which would be consistent with a free-free absorption region partially obscuring the counterjet.

The $8.4 \mathrm{GHz}$ images show that the core, which has been identified spectrally (Jauncey et al. 1995), has a highly inverted spectrum between 4.8 and $8.4 \mathrm{GHz}$ $\left(\alpha=4.1, \mathrm{~S}=\mathrm{k} \nu^{\alpha}\right)$. The core spectral index is clearly above the $\alpha=2.5$ slope predicted by synchrotron self-absorption, and free-free absorption can plausibly explain the excess slope. By making assumptions about the intrinsic spectra of core and pc-scale jet components, we suggest that a free-free absorbing structure exists in the nucleus of Centaurus $\mathrm{A}$, the data being consistent with a torus or thick disk approximately $1 \mathrm{pc}$ in extent, assuming an electron density of $10^{4}$ $\mathrm{cm}^{-3}$ and an electron temperature of $10^{4} \mathrm{~K}$. This absorbing structure appears to partially obscure both jet and counterjet, and seems to be similar to that seen in 3C 84 (Vermeulen, Readhead, \& Backer 1994; Walker, Romney, \& Benson 1994).

\footnotetext{
${ }^{1}$ Current address: Institute of Space and Astronautical Science, Sagamihara, Kanagawa 229, Japan
} 


\section{Constraint on Jet Orientation}

If we define the jet-to-counterjet surface brightness ratio $R$ as the ratio observed at the distance of the first counterjet knot from the core, a value of $R=4$ is found. Alternatively if we use the brightest features in the jet and counterjet, we obtain $R=8$. The most appropriate comparison would be between the smooth underlying jet and counterjet emission but the limited sensitivity of our observations do not allow this. Correcting the observed jet to counterjet brightness ratio for free-free absorption due to the pc- scale torus we get $R$ $=3-7$ for the intrinsic jet-to-counterjet brightness ratio, which provides the constraint $0.15<\beta_{a p p} \cos \theta<0.28$. Combined with the jet speed $\left(\beta_{a p p}>0.45 \mathrm{c}\right)$ inferred from rapid variations in structure and flux density in the structure of the pc-scale jet (Tingay et al. these Proceedings, p. 87), we find that the jet axis of Centaurus $\mathrm{A}$ lies between $50^{\circ}-70^{\circ}$ away from our line of sight. This range is qualitatively consistent with the large-scale morphology of Centaurus A.

Acknowledgments. The Australia Telescope is operated as a national facility by the CSIRO. Part of this work has been undertaken at the Jet Propulsion Laboratory, California Institute of Technology, under contract to the National Aeronautics and Space Administration. Part of this research was conducted while S.J.T. held an NRC-JPL/NASA Research Associateship. The National Radio Astronomy Observatory is a facility of the National Science Foundation, operated under a cooperative agreement by Associated Universities, Inc.

\section{References}

Jauncey, D. L., et al. 1995. Proc. Natl. Acad. Sci. USA, 92, 11368-11370.

Jones, D. L., et al. 1996. ApJ, 466, L63-65.

Preston, R. A., et al. 1996. in IAU Symp. 175, Extragalactic Radio Sources, eds. R. Ekers, C. Fanti, \& L. Padrielli, (Dordrecht: Kluwer), 21-22.

Tingay, S. J., et al. 1996. in Energy Transport in Radio Galaxies and Quasars, eds. P. E. Hardee, A. H. Bridle, \& J. A. Zensus (San Francisco: Astronomical Society of the Pacific), 215-219.

Vermeulen, R. C., Readhead, A. C. S., \& Backer, D. C. 1994. ApJ, 430, L41-44.

Walker, R. C., Romney, J. D., \& Benson, J. M. 1994. ApJ, 430, L45-48. 